\title{
Extinction responding following ICS and food reinforcement'
}

JACK L. CULBERTSON, 2 J. W. KLING AND MARK A. BERKLEY 3

BROWN UNIVERSITY

Rats were trained and extinguished under conditions that were comparable in all respects except for the reinforcement procedures. Food $S s$ responded more frequently during extinction than ICS animals, whether the training had been with CRF or FR-10 reinforcement schedules.

In an attempt to ascertain if behavior reinforced with intracranial stimulation (ICS) differs from more traditionally reinforced behavior, such as food-reinforced behavior, a variety of comparison studies have been undertaken. One such area of research has been concerned with the comparison of resistance to extinction of a response following ICS or food reinforcement. Initially, Olds \& Milner (1954, p. 425) reported that stimulation of the brain "...produces acquisition and extinction curves which compare favorably with those produced by a conventional reward." However, further observations of the persistence of ICS-reinforced responding led to the general impression (cf., Kimble, 1961; Morgan, 1965) that such Ss were less resistant to extinction than food-reinforced animals. One interpretation of these differences in persistence emphasized the variations in the procedures utilized in delivering reinforcement in ICS and food experiments. For example, several studies have examined the effects of modifying the reinforcement contingencies in ICS situations (e.g., Gibson et al, 1965; Pliskoff et al, 1965). In general, these studies conclude that response persistence following ICS reinforcement is not necessarily inferior to that produced by food reinforcement.

The present study was undertaken to determine if differences in free operant responding and subsequent resistance to extinction do in fact exist when food and ICS reinforcement contingencies are not manipulated, but rather, when several other sources of uncontrolled variation are held constant. Some variables which could distort comparisons of food and ICS reinforcement in the typical experiment are the long-term effects of pentobarbital sodium anesthetic on the acquisition and retention of ICS animals (e.g., Stern, 1960); the restraining effects exerted by the electrode lead (Seward et al, 1959); and the differential effectiveness of food-related cues for deprived and non-deprived animals (Campbell \& Sheffield, 1953).

The present study attempted to control the above variables in order to provide a direct comparison of the effects of food and ICS reinforcement procedures. Method

The Ss were 21 naive, male albino rats each weighing approximately $300 \mathrm{gm}$ at the start of the experiment.
Two single-lever boxes, each $9-1 / 4$ in. $x 8-1 / 4$ in. $x$ 19-7/8 in. high, served as the experimental chambers. A rat lever, requiring $12 \mathrm{gm}$ pressure to operate its microswitch, protruded 15/16 in. into each box. Each lever box was housed in a ventilated refrigerator shell, with approximately $70 \mathrm{db}$ (re .0002 dynes $/ \mathrm{cm}^{2}$ ) white noise providing further sound shielding. Illumination was provided by a single 3 watt light bulb. Intracranial stimulation consisted of a 0.5 sec. train of equally spaced biphasic rectangular pulses, with a frequency of $100 \mathrm{pps}$ and a pulse width of $0.2 \mathrm{msec}$. Animals in the Food groups were reinforced with $45 \mathrm{mg}$ Noyes pellets.

All Ss were implanted with bipolar stainless steel electrodes aimed at the posterior portion of the hypothalamus. Following a minimum recovery period of five days all Ss were reduced to $80 \%$ of their ad lib body weights, and maintained at those weights for the remainder of the experiment. Selection of the "optimal" intensity for each ICS animal was determined by shaping bar pressing and then manipulating ICS intensity until rapid responding without interfering motor effects was obtained. Any animal not easily shaped with ICS was assigned to the Food groups and shaped to bar press for food pellets on the following day. Ss preselected to be in the fixed ratio (FR) groups were shaped to the FR-10 schedule of food or ICS reinforcement. Whereas continuous reinforcement (CRF) animals needed an average of one session of bar training, the FR-10 animals needed an average of four sessions. (There were no significant nor suggestive differences between ICS and Food groups in the number of training sessions required to complete the pretraining.) Thereafter each $\mathrm{S}$ received 100 reinforcements a day for 10 successive days. On the day following the session in which the 1,000th reinforcement was obtained, each $S$ entered the first of four daily 15-min. extinction sessions. All Ss were run with electrode leads attached in both the reinforcement and extinction sessions. The number of bar presses emitted and the elapsed time of each session were recorded. The number of animals completing all phases of the experiment was CRF-ICS, $\mathrm{N}=6$; CRF-Food, $\mathrm{N}=5 ;$ FR-ICS, $\mathrm{N}=5 ;$ FR-Food, $\mathrm{N}=5$.

Following completion of the experiment, the animals were sacrificed and histological examinations conducted. Placements were found to be scattered throughout the lateral and posterior portions of the hypothalamus, but no consistent differences in performance were found to be correlated with anatomical locus. Results and Discussion

Figure 1 shows the mean rate of responding for the four groups over the 10 daily reinforcement sessions. 


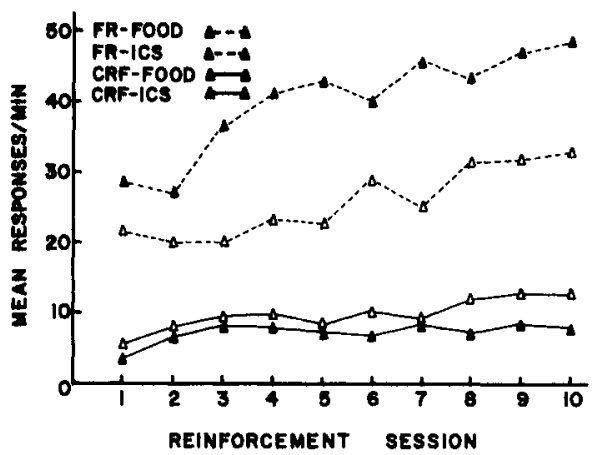

Fig. 1. Mean response rate for the four groups over the 10 daily sessions.

It is obvious that the rates of responding increase over the 10 reinforcement sessions for all four groups, and that there are stable between-group response rate differences. A Lindquist (1953) Type III analysis of variance was performed on this data. Because of the unequal number of Ss in the groups, the Lindquist procedure for missing cases was utilized. This analysis revealed a significant Days (D) effect ( $F=19.3$; df $=9 / 153$; $p<$ $.001)$, a significant Schedules (S) effect ( $F=41.6$; $\mathrm{df}=1 / 17 ; \mathrm{p}<.001$ ), but no significant Reinforcer $(R)$ effect $(F=2.8 ; d f=1 / 17 ; p<.20)$. This analysis also revealed a significant $D$ by $S$ interaction $(F=5.8$; df $=9 / 153 ; p<.001$ ) which reflects the fact that the CRF groups show little increase in rate after the first few sessions, whereas both FR groups continue to accelerate over all 10 sessions. A significant $D$ by $R$ interaction $(F=2.7 ; d f=9 / 153 ; p<.01)$ as well as a significant $R$ by $S$ interaction $(F=7.7 ; d f=1 / 17 ; p<.025)$ were also revealed by the analysis. The $R$ by $S$ interaction can be seen in Fig. 1, in that the FR-ICS Ss respond more slowly than the FR-Food Ss, whereas in CRF the ICS animals respond faster than the Food Ss. This may reflect both the time lost by the Food animals in eating and a genuine superiority of Food animals on intermittent schedules.

Figure 2 shows the mean number of extinction responses emitted by each group over the four daily sessions. An examination of this figure reveals the following results: the number of extinction responses emitted decreases daily for all four groups, the Food groups emit a greater number of extinction responses than the ICS groups, and the FR groups emit more extinction responses than the CRF groups. It is interesting to note that although the type of reinforcer appears to be an effective parameter in determining the amount of extinction responding, the schedule of reinforcement (at least in the present study) seems to be an even more potent determinant of response persistence. A Lindquist Type III analysis of variance was performed on the extinction data using the missing cases procedure. Because

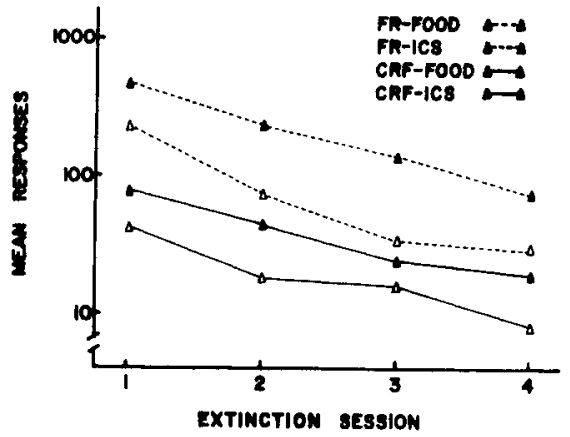

Fig. 2. Mean number of response emitted during the four daily extinction sessions.

the variance within groups was linearly related to the magnitude of the means, a logarithmic transformation was utilized in the analysis. The analysis of variance confirmed a significant Reinforcer effect ( $F=27.5$; df = $1 / 17 ; p<.001)$, a significant Schedule effect $(F=67.1$; $\mathrm{df}=1 / 17 ; \mathrm{p}<.001)$, and a significant Days effect $(\mathrm{F}=93.9$; $\mathrm{df}=3 / 51 ; \mathrm{p}<.001$ ).

The general forms of the acquisition and extinction curves are remarkably similar for Food and ICS groups, but the results do confirm the general impression that food reinforcement will produce the somewhat greater resistance to extinction when all but the reinforcement conditions are held constant. The results also suggest that these differences will be found even if varying degrees of response persistence are established through the use of different reinforcement schedules.

\section{References}

Campbell, B. A., \& Sheffield, F. D. Relation of random activity to food deprivation. J. comp. physiol. Psychol, 1953, 46, 320-322.

Gibson, W. E., Reid, L. D.. Sakai, M., \& Porter, P. B. Intracranial teinforcement compared with sugar-water reinforcement. Science, $1965,148,1357-1359$.

Kimble, G. A. Hilgard and Marquis' conditioning and learning. New York: Appleton-Century-Crofts, 1961. P. 263.

Lindquist, E. F. Design and analysis of experiments in psychology and education. Boston: Houghton Mifflin, 1953.

Morgan, C. T. Physiological psychology. New York: McGraw-Hill, 1965. P. 329.

Olds, J., \& Milner, P. Positive reinforcement produced by electrical stimulation of septal area and other regions of rat brain. J. comp. physiol. Psychol., 1954, 47, 419-427.

Pliskoff, S. S., Wright, J. E., \& Hawkins, T. D. Brain stimulation as a reinforcer: Intermittent schedules. J. exp. Anal. Behav., $1965,8,75-88$.

Seward, J. P., Uyeda, A. A., \& Olds, J. Resistance to extinction following cranial self-stimulation. J. comp. physiol. Psychol., 1959, 52, 294-299.

Stem, Muriel h. Behavior retardation following barbiturate anesthesia. Canad. J. Psychol., 1960, 14, 96-100.

\section{Notes}

1. This research was supported by USPHS Grant No. MH- 05767 .

2. USPHS Pre-doctoral Fellow.

3. Now at Department of Physiology and Biophysics, University of Washington. 